Article

\title{
On approximation methods in some geodesic spaces without the nice projection property
}

\author{
Pongsakorn Yotkaew \\ Department of Mathematics, Faculty of Science, Khon Kaen University, Khon Kaen 40002, Thailand; \\ pongyo@kku.ac.th
}

\begin{abstract}
The purpose of this paper is to prove strong convergent theorems for Browder's type iterations and Halpern's type iterations of a family of nonexpansive mappings in a complete geodesic space with curvature bounded above by a positive number. Moudafi's viscosity type methods are also discussed without the nice projection property.
\end{abstract}

Keywords: Browder's type iteration; CAT(1) space; fixed point; Halpern's type iteration; Moudafi's viscosity type method; nonexpansive mapping

MSC: $47 \mathrm{H} 09 ; 47 \mathrm{H} 10$

\section{Introduction}

One of the most important analytical problems is the existence of fixed points for nonlinear mappings. The well-known Banach contraction principle guarantees that every contraction on a complete metric space has a unique fixed point. The existence of fixed point theorems for a nonexpansive mapping in CAT $(\kappa)$ spaces was proved by Kirk [1,2] for $\kappa \leq 0$, and by Espánola and Fernández-León [3] for $\kappa>0$. Nevertheless, any CAT $\left(\kappa^{\prime}\right)$ space is a CAT $(\kappa)$ space for $\kappa^{\prime}<\kappa$, so all results of $\operatorname{CAT}(0)$ spaces can immediately be applied to any $\operatorname{CAT}(\kappa)$ with $\kappa \leq 0$. Moreover, $\operatorname{CAT}(\kappa)$ spaces with positive $\kappa$ can be treated as CAT(1) spaces by changing the scale of the space; see $[4,5]$ for more details. It therefore suffices to focus only on CAT(1) spaces.

There are several methods for approximating fixed points of a nonexpansive mapping $T$ on a complete CAT(1) space $X$ such that $\mathrm{F}(T):=\{x \in X: x=T x\} \neq \varnothing$. In 2011, Piastek presented the following result.

Theorem 1.1 ([6, Theorem 3.5]). Let $u \in X$ be fixed and suppose that $d(u, \mathrm{~F}(T)) \leq \pi / 4$. Denote $q:=P_{\mathrm{F}(T)} u$, where $P_{\mathrm{F}(T)}$ is the projection mapping from $X$ onto $\mathrm{F}(T)$. Then for each $t \in(0,1)$, there exists the unique fixed point $x_{t} \in \bar{B}(q, \pi / 4)$ of the contraction $x \mapsto t f(x) \oplus(1-t)$ Tx on $\bar{B}(q, \pi / 4)$, that is,

$$
x_{t}=t u \oplus(1-t) T x_{t}
$$

In addition, the net $\left\{x_{t}\right\}$ converges to the unique fixed point $q$ of $T$ which is nearest to $u$ as $t \rightarrow 0^{+}$.

In 2013, Kimura and Satô proved the following convergence theorem of Halpern's type iterations in a complete CAT(1) space $X$.

Theorem 1.2 ([7, Theorem 5.5]). Let $X$ be a complete $C A T(1)$ space such that $d(v, w)<\pi / 2$ for all $v$, $w \in X$, and $T$ be a nonexpansive mapping on $X$ such that $\mathrm{F}(T) \neq \varnothing$. Let $\left\{x_{n}\right\}$ be the sequence in $X$ generated by $x_{1}=u \in X$ and

$$
x_{n+1}:=\alpha_{n} u \oplus\left(1-\alpha_{n}\right) T x_{n} \quad \text { for } n \in \mathbb{N},
$$


where $\left\{\alpha_{n}\right\}$ is a sequence in $[0,1]$ such that $\lim _{n \rightarrow \infty} \alpha_{n}=0$ and $\sum_{n=1}^{\infty} \alpha_{n}=\infty$. Suppose that one of the following conditions holds:

(a) $\sup \{d(v, w): v, w \in X\}<\pi / 2$;

(b) $\sum_{n=1}^{\infty} \alpha_{n}^{2}=\infty$.

Then the sequence $\left\{x_{n}\right\}$ converges to the unique fixed point $q$ of $T$ which is nearest to $u$.

In 2016, Huang [8] presented the following convergence result of Browder's type iterations for a family of nonexpansive mappings in a complete CAT(0) space.

Theorem 1.3 ([8, Theorem 4.5]). Let $S$ be a nonexpansive mapping on a complete $C A T(0)$ space $X$, and $\left\{T_{n}\right\}$ be a family of nonexpansive mappings on $X$ such that $\varnothing \neq \operatorname{Fix}(S)=\bigcap_{n=1}^{\infty} \operatorname{Fix}\left(T_{n}\right)$. Let $\left\{x_{n}\right\}$ be the sequence in $X$ generated by

$$
x_{n}=\alpha_{n} u \oplus\left(1-\alpha_{n}\right) T_{n} x_{n} \quad \text { for } n \in \mathbb{N},
$$

where $\left\{\alpha_{n}\right\}$ is a sequence in $[0,1]$ such that $\lim _{n \rightarrow \infty} \alpha_{n}=0$. If $\left(\left\{T_{n}\right\}, S\right)$ satisfies the NST-condition, then the sequence $\left\{x_{n}\right\}$ converges strongly to the point $P_{\mathrm{F}(S)} u$.

In 2012, Shi and Chen [9] presented a convergence result of Moudafi's viscosity type iterations for a nonexpansive mapping $T$ on a complete CAT(0) space $X$ such that $\mathrm{F}(T) \neq \varnothing$. To be precise, for a contraction $f$ on $X$ and $t \in(0,1)$, there exists the unique fixed point $x_{t} \in X$ of the contraction $x \mapsto t f(x) \oplus(1-t) T x$, that is,

$$
x_{t}=t f\left(x_{t}\right) \oplus(1-t) T x_{t} .
$$

Moreover, the net $\left\{x_{t}\right\}$ converges to $q:=P_{\mathrm{F}(T)} f(q)$ as $t \rightarrow 0^{+}$in the framework of the space satisfying the nice projection property, i.e., for all $u, v, w_{1}, w_{2} \in X$, we have

$$
d\left(v, P_{\left[v, w_{1}\right]} u\right) d\left(v, w_{1}\right) \leq d\left(x, P_{\left[v, w_{2}\right]} u\right) d\left(v, w_{2}\right)+d(v, u) d\left(w_{1}, w_{2}\right) .
$$

Next, Wangkeeree and Preechasilp [10] improved the above results of Shi and Chen without assuming the nice projection property. Since then, many authors have worked on Moudafi's viscosity type iterations for different types of mappings on CAT(0) spaces; see, e.g., [11-15]. Unfortunately, the result of Shi and Chen are still unknown in the framework of a CAT(1) space without the nice projection property.

In this paper, we prove an existence and convergence theorem which supplements Theorem 1.1 of Piątek. Furthermore, we present a strong convergent theorem for Browder's type iterations of a family of nonexpansive mappings, which extends Theorem 1.3 of Huang from CAT(0) spaces to CAT(1) spaces. Next, we derive our results with Halpern's type iteration of a family of nonexpansive mappings, which extends Theorem 1.2 of Kimura and Satô. Finally, Moudafi's viscosity type methods are also discussed without the nice projection property.

\section{Some properties of CAT(1) spaces}

Let $(X, d)$ be a CAT(1) space such that $d(v, w)<\pi$ for all $v, w \in X$. Given points $v, w \in X$ and $t \in[0,1]$, we use the notation $t v \oplus(1-t) w$ for the unique point $u$ in the geodesic segment joining $v$ and $w$ such that

$$
d(u, v)=(1-t) d(v, w) \text { and } d(u, w)=t d(v, w) .
$$

The following lemma yields a crucial inequality in CAT(1) spaces, which plays an important role in this paper. 
Lemma 2.1 ([16, Corollary 2.2]). Let $t \in[0,1]$ and $u, v, w$ be three points in a CAT $(1)$ space $(X, d)$ such that $d(u, v)+d(v, w)+d(w, u)<2 \pi$. Then

$$
\begin{aligned}
\cos d(t v \oplus(1-t) w, u) \sin d(v, w) \geq & \cos d(v, u) \sin (t d(v, w)) \\
& +\cos d(w, u) \sin ((1-t) d(v, w)) .
\end{aligned}
$$

Let $C$ be a closed convex subset of a complete $\operatorname{CAT}(1)$ space $(X, d)$ such that $d(v, C):=$ $\inf _{w \in C} d(v, w)<\pi / 2$ for all $v \in X$. Then the metric projection $P_{C}$ from $X$ onto $C$ is well defined; that is, for each $v \in X$, there exists the unique point $P_{C} v \in C$ satisfying

$$
d\left(v, P_{C} v\right)=\inf _{w \in C} d(v, w)
$$

Using the preceding lemma, we can now present the following characterization of the metric projection in CAT(1) spaces.

Proposition 2.2. Let $(X, d)$ be a CAT(1) space such that $d(v, w)<\pi / 2$ for all $v, w \in X$, and let $C$ be a nonempty closed convex subset of $X$. If $u \in X$ and $w \in C$, then

$$
w=P_{C} u \text { if and only if } \cos d(u, v) \leq \cos d(u, w) \cos d(v, w) \text { for all } v \in C,
$$

where $P_{C}$ is the metric projection from $X$ onto $C$.

Proof. Assume first that $\cos d(u, v) \leq \cos d(u, w) \cos d(v, w)$ for all $v \in C$. It follows that $d(u, w) \leq$ $d(u, v)$ for all $v \in C$. Then we conclude that $w=P_{C} u$.

Conversely, assume that $w=P_{C} u$ and let $v \in C$. If $v=w$, then the result follows. Now, suppose that $v \neq w$. For each $t \in(0,1)$, by the convexity of $C$, we have $t v \oplus(1-t) w \in C$. This implies that $d(u, w) \leq d(u, t v \oplus(1-t) w)$. It follows from Lemma 2.1 that

$$
\begin{aligned}
\cos d(u, w) & \geq \cos d(u, t v \oplus(1-t) w) \\
& \geq \cos d(u, v) \frac{\sin t d(v, w)}{\sin d(v, w)}+\cos d(u, w) \frac{\sin (1-t) d(v, w)}{\sin d(v, w)}
\end{aligned}
$$

Hence, we have

$$
\begin{aligned}
0 & \leq \cos d(u, w)\left(1-\frac{\sin (1-t) d(v, w)}{\sin d(v, w)}\right)-\cos d(u, v) \frac{\sin t d(v, w)}{\sin d(v, w)} \\
& =\cos d(u, w) \frac{2 \sin (t / 2) d(v, w) \cos (1-t / 2) d(v, w)}{\sin d(v, w)}-\cos d(u, v) \frac{\sin t d(v, w)}{\sin d(v, w)} .
\end{aligned}
$$

Dividing both sides of the above inequality by $t$ and letting $t$ tend to 0 , we obtain

$$
0 \leq(\cos d(u, w) \cos d(v, w)-\cos d(u, v)) \frac{d(v, w)}{\sin d(v, w)}
$$

Consequently, we have $\cos d(u, v) \leq \cos d(u, w) \cos d(v, w)$, and the proof is finished.

Lemma 2.3 ([17, Lemma 3.10]). Let $v_{0}, v_{1}, w_{0}, w_{1}$ be four elements in a CAT(1) space $(X, d)$ such that $d(v, w)<\pi / 2$ for all $v, w \in\left\{v_{0}, v_{1}, w_{0}, w_{1}\right\}$. Then

$$
\cos d\left(v_{0}, w_{1}\right)+\cos d\left(v_{1}, w_{0}\right)-\cos d\left(v_{0}, v_{1}\right)-\cos d\left(w_{0}, w_{1}\right) \leq d\left(v_{0}, w_{0}\right) d\left(v_{1}, w_{1}\right) .
$$


In CAT(1) spaces, it is known that the metric projection is a Lipschitz mapping although it may be expansive [18]. The following lemma can be deduced from the proof of [17, Theorem 4.1] together with the above lemma.

Lemma 2.4. Let $X$ be a complete $C A T(1)$ space such that $M:=\sup \{d(v, w): v, w \in X\}<\pi / 2$, and let $C$ be a nonempty closed convex subset of $X$. Then

$$
d\left(P_{C} v, P_{C} w\right) \leq \frac{M^{2}}{4 \sin ^{2}\left(\frac{M}{2}\right) \cos M} d(v, w)
$$

for all $v, w \in X$, where $P_{C}$ is the metric projection from $X$ onto $C$.

Proof. Let $v, w \in X$. By Lemma 2.2, we have

$$
\left.\cos d\left(P_{C} v, w\right) \leq \cos d\left(w, P_{C} w\right) \cos d\left(P_{C} v\right), P_{C} w\right)
$$

and

$$
\left.\cos d\left(P_{C} w, v\right) \leq \cos d\left(v, P_{C} v\right) \cos d\left(P_{C} v\right), P_{C} w\right) .
$$

It follows from Lemma 2.3 that

$$
\begin{aligned}
& d(v, w) d\left(P_{C} v, P_{C} w\right) \\
& \geq \cos d\left(P_{F} v, v\right)+\cos d\left(P_{F} w, w\right)-\cos d\left(P_{F} v, w\right)-\cos d\left(P_{F} w, v\right) \\
& \left.\geq \cos d\left(P_{F} v, v\right)+\cos d\left(P_{F} w, w\right)-\cos d\left(w, P_{F} w\right) \cos d\left(P_{F} v\right), P_{F} w\right) \\
& \left.\quad-\cos d\left(v, P_{F} v\right) \cos d\left(P_{F} v\right), P_{F} w\right) \\
& =\left(\cos d\left(P_{F} v, v\right)+\cos d\left(P_{F} w, w\right)\right)\left(1-\cos d\left(P_{C} v, P_{C} w\right)\right) \\
& =\left(\cos d\left(P_{F} v, v\right)+\cos d\left(P_{F} w, w\right)\right) 2 \sin ^{2}\left(\frac{d\left(P_{C} v, P_{C} w\right)}{2}\right) \\
& \geq 4 \cos M \sin ^{2}\left(\frac{M}{2}\right)\left(\frac{d\left(P_{C} v, P_{C} w\right)}{M}\right)^{2} .
\end{aligned}
$$

Consequently, we have

$$
d\left(P_{C} v, P_{C} w\right) \leq \frac{M^{2}}{4 \sin ^{2}\left(\frac{M}{2}\right) \cos M} d(v, w),
$$

and the proof is finished.

The following lemmas are required for our main results.

Lemma 2.5 ([7, Lemma 5.4]). Let $u, v, w$ be three points in a $\operatorname{CAT}(1)$ space $(X, d)$ such that $d(u, v)+$ $d(v, w)+d(w, u)<2 \pi$. Let $x=t u \oplus(1-t) v$ and $y=t u \oplus(1-t) w$ for some $t \in[0,1]$. If $d(u, v) \leq M$, $d(u, w) \leq M$, and $\sin ((1-t) M) \leq \sin M$ for some $M \in(0, \pi)$, then

$$
d(x, y) \leq \frac{\sin (1-t) M}{\sin M} d(v, w) .
$$

Lemma 2.6 ([19, Lemma 2.3]). Let $u, v, w$ be three points in a CAT $(1)$ space $(X, d)$ such that $d(v, u) \leq \pi / 2$ and $d(w, u) \leq \pi / 2$, and let $t \in[0,1]$. Then

$$
\cos d(t v \oplus(1-t) w, u) \geq t \cos d(v, u)+(1-t) \cos d(w, u) .
$$

Moreover, we have $d(t v \oplus(1-t) w, u) \leq \max \{d(v, u), d(w, u)\}[20$, Lemma 3.4]. 
Lemma 2.7 ([21, Lemma 2.5]). Let $\left\{a_{n}\right\}$ and $\left\{c_{n}\right\}$ be sequences of nonnegative real numbers, $\left\{b_{n}\right\}$ be a sequence of real numbers, and $\left\{\gamma_{n}\right\}$ be a sequence of real numbers in $[0,1]$ such that

$$
a_{n+1} \leq\left(1-\gamma_{n}\right) a_{n}+\gamma_{n} b_{n}+c_{n}
$$

for all $n \in \mathbb{N}$. If $\lim \sup _{n \rightarrow \infty} b_{n} \leq 0, \sum_{n=1}^{\infty} c_{n}<\infty$, and $\sum_{n=1}^{\infty} \gamma_{n}=\infty$, then $\lim _{n \rightarrow \infty} a_{n}=0$.

Let $\ell_{\infty}$ denote the Banach space of bounded real sequences. Recall that a continuous linear functional $\mu$ on $\ell_{\infty}$ is said to be a Banach limit if $\|\mu\|=\mu(1,1, \ldots)=1$ and $\mu_{n}\left(a_{n}\right)=\mu_{n}\left(a_{n+1}\right)$ for all $\left\{a_{n}\right\} \in \ell_{\infty}$.

Lemma 2.8 ([22, Proposition 2]). Let $\left\{a_{n}\right\} \in \ell_{\infty}$ be such that $\mu_{n}\left(a_{n}\right) \leq 0$ for all Banach limits $\mu$. If $\limsup _{n \rightarrow \infty}\left(a_{n+1}-a_{n}\right) \leq 0$, then $\lim \sup _{n \rightarrow \infty} a_{n} \leq 0$.

\section{Strong convergence theorem for Browder's type}

Throughout the rest of this paper, let $X$ denote a complete CAT(1) space with $d(v, w)<\pi / 2$ for all $v, w \in X$ and $M:=\sup \{d(v, w): v, w \in X\}$ be the diameter of $X$. Recall that a mapping $T: X \rightarrow X$ is nonexpansive if

$$
d(T v, T w) \leq d(v, w) \text { for all } v, w \in X
$$

A sequence $\left\{v_{n}\right\} \subset X$ is said to be an approximating fixed point sequence of $T$ if

$$
\lim _{n \rightarrow \infty} d\left(v_{n}, T v_{n}\right)=0
$$

The approximating fixed point sequence plays an important role in the study of fixed point theory of nonexpansive mappings. We begin with the existence and some properties of approximating fixed point sequences of nonexpansive mappings.

Proposition 3.1. Let $T: X \rightarrow X$ be a nonexpansive mapping and $u \in X$ be fixed. Given a point $t \in(0,1)$, define $S_{t}: X \rightarrow X$ by

$$
S_{t} x=t u \oplus(1-t) T x \quad \text { for } x \in X .
$$

Then $S_{t}$ has a unique fixed point $x_{t} \in X$, that is,

$$
x_{t}=t u \oplus(1-t) T x_{t}
$$

In this case, we have $d\left(x_{t}, T x_{t}\right) \rightarrow 0$ as $t \rightarrow 0^{+}$. Moreover, the following statements hold:

1. If $0<s<t<1$, then

$$
\cos d\left(u, x_{s}\right) \leq \cos d\left(u, x_{t}\right) \cos d\left(x_{t}, x_{s}\right)
$$

2. If $\mathrm{F}(T) \neq \varnothing$, then

$$
\cos d(u, p) \leq \cos d\left(u, x_{t}\right) \cos d\left(x_{t}, p\right)
$$

for all $p \in \mathrm{F}(T)$ and $t \in(0,1)$.

Proof. Given points $x, y \in X$ and $t \in(0,1)$, by Lemma 2.5, we obtain

$$
\begin{aligned}
d\left(S_{t} x, S_{t} y\right) & =d(t u \oplus(1-t) T x, t u \oplus(1-t) T y) \\
& \leq\left(\sin \frac{(1-t) \pi}{2}\right) d(T x, T y) \\
& \leq\left(\sin \frac{(1-t) \pi}{2}\right) d(x, y)
\end{aligned}
$$


Then $S_{t}$ is a contraction. It follows from the Banach contraction principle that there exists exactly one point $x_{t} \in X$ such that

$$
x_{t}=t u \oplus(1-t) T x_{t}
$$

Moreover, we have

$$
d\left(x_{t}, T x_{t}\right)=t d\left(u, T x_{t}\right) \text { and } d\left(u, x_{t}\right)=(1-t) d\left(u, T x_{t}\right) .
$$

This implies that

$$
\lim _{t \rightarrow 0^{+}} d\left(x_{t}, T x_{t}\right)=\lim _{t \rightarrow 0^{+}} \frac{t}{1-t} d\left(u, x_{t}\right)=0 .
$$

To prove the statement $(1)$, let $s, t \in(0,1)$ be such that $s<t$. Consider a comparison triangle $\triangle\left(\bar{u}, \overline{T x_{s}}, \overline{T x_{t}}\right)$ for $\triangle\left(u, T x_{s}, T x_{t}\right)$ in $\mathbb{S}^{2}$. Put $z_{s}:=s u \oplus(1-s) T x_{t}$. It is obvious that $z_{s}$ lies in $\left[x_{t}, T x_{t}\right]$. Applying Lemma 2.5 with $M=\pi / 2$, we obtain

$$
\begin{aligned}
d\left(\overline{x_{s}}, \overline{z_{s}}\right) & =d\left(s \bar{u} \oplus(1-s) \overline{T x_{s}}, s \bar{u} \oplus(1-t) \overline{T x_{t}}\right) \\
& \leq d\left(\overline{T x_{t}}, \overline{T x_{s}}\right)=d\left(T x_{s}, T x_{t}\right) \leq d\left(x_{s}, x_{t}\right) \leq d\left(\overline{x_{s}}, \overline{x_{t}}\right) .
\end{aligned}
$$

This together with the spherical law of cosines for $\triangle\left(\overline{x_{s}}, \overline{x_{t}}, \overline{z_{s}}\right)$ yields

$$
\begin{aligned}
0 & \leq \cos d\left(\overline{x_{s}}, \overline{z_{s}}\right)-\cos d\left(\overline{x_{s}}, \overline{x_{t}}\right) \\
& \leq \cos d\left(\overline{x_{s}}, \overline{z_{s}}\right)-\cos d\left(\overline{x_{s}}, \overline{x_{t}}\right) \cos d\left(\overline{x_{t}}, \overline{z_{s}}\right) \\
& =\sin d\left(\overline{x_{s}}, \overline{x_{t}}\right) \sin d\left(\overline{x_{t}}, \overline{z_{s}}\right) \cos \angle \overline{x_{t}}\left(\overline{x_{s}}, \overline{z_{s}}\right),
\end{aligned}
$$

where $\angle \overline{x_{t}}\left(\overline{x_{s}}, \overline{z_{s}}\right)$ is the angle between edges $\left[\overline{x_{t}}, \overline{x_{s}}\right]$ and $\left[\overline{x_{t}}, \overline{z_{s}}\right]$. This implies that $\angle \overline{x_{t}}\left(\overline{x_{s}}, \overline{z_{s}}\right) \leq \pi / 2$, and hence

$$
\angle \overline{x_{t}}\left(\overline{x_{s}}, \bar{u}\right)=\pi-\angle \overline{x_{t}}\left(\overline{x_{s}}, \overline{z_{s}}\right) \geq \pi / 2
$$

Consequently, we have

$$
\begin{aligned}
\cos d\left(u, x_{s}\right) & =\cos d\left(\bar{u}, \overline{x_{s}}\right) \\
& =\cos d\left(\bar{u}, \overline{x_{t}}\right) \cos d\left(\overline{x_{t}}, \overline{x_{s}}\right)+\sin d\left(\bar{u}, \overline{x_{t}}\right) \sin d\left(\overline{x_{t}}, \overline{x_{s}}\right) \cos \angle \overline{x_{t}}\left(\overline{x_{s}}, \bar{u}\right) \\
& \leq \cos d\left(\bar{u}, \overline{x_{t}}\right) \cos d\left(\overline{x_{t}}, \overline{x_{s}}\right) \\
& \leq \cos d\left(u, x_{t}\right) \cos d\left(x_{t}, x_{s}\right)
\end{aligned}
$$

To verify the statement (2), fix $p \in \mathrm{F}(T)$ and $t \in(0,1)$. Consider a comparison triangle $\triangle\left(\bar{u}, \bar{p}, \overline{T x_{t}}\right)$ for $\triangle\left(u, p, T x_{t}\right)$ in $\mathbb{S}^{2}$. The nonexpansiveness of $T$ implies that

$$
d\left(\bar{p}, \overline{T x_{t}}\right)=d\left(p, T x_{t}\right) \leq d\left(p, x_{t}\right) \leq d\left(\bar{p}, \overline{x_{t}}\right)
$$

In a similar way to the proof of the first statement, by the spherical law of cosines for $\triangle\left(\bar{p}, \overline{x_{t}}, \overline{T x_{t}}\right)$, we obtain

$$
\angle \overline{x_{t}}(\bar{p}, \bar{u})=\pi-\angle \overline{x_{t}}\left(\bar{p}, \overline{T x_{t}}\right) \geq \pi / 2 .
$$

As a result, we have $\cos d(u, p) \leq \cos d\left(u, x_{t}\right) \cos d\left(x_{t}, p\right)$. This completes the proof.

As an immediate consequence of Proposition 3.1, we obtain the following result.

Corollary 3.2. Every nonexpansive mapping $T: X \rightarrow X$ has approximating fixed point sequences.

The statement (1) of the following theorem was proved by Piatek in [6] under the condition $d(u, \mathrm{~F}(T)) \leq \pi / 4$ (see Theorem 1.1). We present here another version of its proof which is modified from Saejung's proof in [23] under suitable conditions. 
Theorem 3.3. Let $X, T, u$ be as in the preceding proposition and let $\left\{x_{t}\right\}$ be a net given by the formula (3.1). Then $\mathrm{F}(T) \neq \varnothing$ if and only if

$$
\operatorname{rad}_{X}\left(\left\{x_{t}\right\}\right):=\inf _{z \in X} \sup _{t \in(0,1)} d\left(x_{t}, z\right)<\pi / 2
$$

In this case, the following statements hold:

1. The net $\left\{x_{t}\right\}$ converges to a unique fixed point $q$ of $T$ which is nearest to $u$ as $t \rightarrow 0^{+}$.

2. For all Banach limits $\mu$ and all approximating fixed point sequences $\left\{y_{n}\right\}$ of $T$, we have $\cos d(u, q) \geq$ $\mu_{n} \cos d\left(u, y_{n}\right)$.

Proof. Suppose first that $\mathrm{F}(T) \neq \varnothing$. Fix $p \in \mathrm{F}(T)$ and let $t \in(0,1)$ be an arbitrary element. By Lemma 2.6, we have

$$
\begin{aligned}
\cos d\left(x_{t}, p\right) & =\cos d\left(t u \oplus(1-t) T x_{t}, p\right) \\
& \geq t \cos d(u, p)+(1-t) \cos d\left(T x_{t}, p\right) \\
& \geq t \cos d(u, p)+(1-t) \cos d\left(x_{t}, p\right) .
\end{aligned}
$$

This implies that $\cos d\left(x_{t}, p\right) \geq \cos d(u, p)$. It follows that $d\left(x_{t}, p\right) \leq d(u, p)<\pi / 2$, and hence $\operatorname{rad}_{X}\left(\left\{x_{t}\right\}\right)<\pi / 2$

Conversely, suppose that $\operatorname{rad}_{X}\left(\left\{x_{t}\right\}\right)<\pi / 2$. Let $\left\{t_{k}\right\}$ be any sequence in $(0,1)$ such that $\lim _{k \rightarrow \infty} t_{k}=0$ and define $g: X \rightarrow[0,1]$ by

$$
g(z)=\liminf _{k \rightarrow \infty} \cos d\left(x_{t_{k}}, z\right) \quad \text { for } z \in X
$$

By the assumption, we have $A:=\sup \{g(z): z \in X\}>0$. Let $\left\{z_{n}\right\}$ be a sequence in $X$ such that $\lim _{n \rightarrow \infty} g\left(z_{n}\right)=A$. For $m, n \in \mathbb{N}$, if $z_{m}=z_{n}$, then we obtain

$$
\cos \frac{1}{2} d\left(z_{m}, z_{n}\right) \geq \frac{1}{A}\left(\frac{1}{2} g\left(z_{m}\right)+\frac{1}{2} g\left(z_{n}\right)\right) .
$$

On the other hand, if $z_{m} \neq z_{n}$, then we have

$$
\begin{aligned}
& \cos d\left(x_{t_{k}}, \frac{1}{2} z_{m} \oplus \frac{1}{2} z_{n}\right) \\
& \geq \cos d\left(x_{t_{k}}, z_{m}\right) \frac{\sin \frac{1}{2} d\left(z_{m}, z_{n}\right)}{\sin d\left(z_{m}, z_{n}\right)}+\cos d\left(x_{t_{k}}, z_{n}\right) \frac{\sin \frac{1}{2} d\left(z_{m}, z_{n}\right)}{\sin d\left(z_{m}, z_{n}\right)} \\
& =\left(\frac{1}{2} \cos d\left(x_{t_{k}}, z_{m}\right)+\frac{1}{2} \cos d\left(x_{t_{k}}, z_{n}\right)\right) \frac{1}{\cos \frac{1}{2} d\left(z_{m}, z_{n}\right)}
\end{aligned}
$$

for all $k$. Thus, if we take the limit inferior to both sides of the above inequality as $k \rightarrow \infty$, we obtain

$$
\begin{aligned}
A & \geq \liminf _{k \rightarrow \infty} \cos d\left(x_{t_{k}}, \frac{1}{2} z_{m} \oplus \frac{1}{2} z_{n}\right) \\
& \geq \liminf _{k \rightarrow \infty}\left(\frac{1}{2} \cos d\left(x_{t_{k}}, z_{m}\right)+\frac{1}{2} \cos d\left(x_{t_{k}}, z_{n}\right)\right) \frac{1}{\cos \frac{1}{2} d\left(z_{m}, z_{n}\right)} \\
& \geq\left(\frac{1}{2} \liminf _{k \rightarrow \infty} \cos d\left(x_{t_{k}}, z_{m}\right)+\frac{1}{2} \liminf _{k \rightarrow \infty} \cos d\left(x_{t_{k}}, z_{n}\right)\right) \frac{1}{\cos \frac{1}{2} d\left(z_{m}, z_{n}\right)} \\
& =\left(\frac{1}{2} g\left(z_{m}\right)+\frac{1}{2} g\left(z_{n}\right)\right) \frac{1}{\cos \frac{1}{2} d\left(z_{m}, z_{n}\right)}
\end{aligned}
$$


and hence

$$
\cos \frac{1}{2} d\left(z_{m}, z_{n}\right) \geq \frac{1}{A}\left(\frac{1}{2} g\left(z_{m}\right)+\frac{1}{2} g\left(z_{n}\right)\right) .
$$

Since $\lim _{n \rightarrow \infty} g\left(z_{n}\right)=A$, it follows that

$$
\lim _{m, n \rightarrow \infty} \cos \frac{1}{2} d\left(z_{m}, z_{n}\right)=1 .
$$

As a result, the sequence $\left\{z_{n}\right\}$ is a Cauchy sequence in $X$. Then it converges to a point $\widehat{z} \in X$ and it is easy to check that $g(\widehat{z})=A$. Suppose that $\bar{z}$ is a point in $X$ satisfying $g(\bar{z})=A$. In the same way, one can replace $\left(z_{m}, z_{n}\right)$ with $(\widehat{z}, \bar{z})$ in the above equation, that is,

$$
\cos \frac{1}{2} d(\widehat{z}, \bar{z})=1
$$

Consequently, we have $\bar{z}=\widehat{z}$ and also $\widehat{z} \in \mathrm{F}(T)$. In fact, Theorem 3.3 implies that $d\left(x_{t_{k}}, T x_{t_{k}}\right) \rightarrow 0$ as $k \rightarrow \infty$. It then follows that

$$
\begin{aligned}
A & \geq \liminf _{k \rightarrow \infty} \cos d\left(x_{t_{k}}, T \widehat{z}\right) \\
& \geq \liminf _{k \rightarrow \infty} \cos \left(d\left(x_{t_{k}}, T x_{t_{k}}\right)+d\left(T x_{t_{k}}, T \widehat{z}\right)\right) \\
& \geq \liminf _{k \rightarrow \infty} \cos \left(d\left(x_{t_{k}}, T x_{t_{k}}\right)+d\left(x_{t_{k}}, \widehat{z}\right)\right) \\
& =\liminf _{k \rightarrow \infty} \cos d\left(x_{t_{k}}, \widehat{z}\right)=A,
\end{aligned}
$$

and hence $g(T \widehat{z})=A$. Thus, we can conclude that $\widehat{z}$ is a fixed point of $T$.

Next, we will verify the statement (1). For $s, t$, such that $0<s<t<1$, by Proposition 3.1, we have

$$
\cos d\left(u, x_{s}\right) \leq \cos d\left(u, x_{t}\right) \cos d\left(x_{t}, x_{s}\right) \leq \cos d\left(u, x_{t}\right)
$$

and

$$
\cos d(u, \widehat{z}) \leq \cos d\left(u, x_{t}\right) \cos d\left(x_{t}, \widehat{z}\right) \leq \cos d\left(u, x_{t}\right) .
$$

Therefore, the net $\left\{d\left(u, x_{t}\right)\right\}$ is monotonically decreasing and

$$
\lim _{t \rightarrow 0^{+}} d\left(u, x_{t}\right) \leq d(u, \widehat{z})<\frac{\pi}{2}
$$

Consequently, we have

$$
\cos d\left(x_{t}, x_{s}\right) \geq \frac{\cos d\left(u, x_{s}\right)}{\cos d\left(u, x_{t}\right)} \rightarrow 1 \text { as } s, t \rightarrow 0^{+} .
$$

It follows directly from the above inequality that $\left\{x_{t}\right\}$ converges to a point $q \in X$ and it is easy to check that $q$ is a fixed point of $T$. Furthermore, one can see $q$ is the point of $\mathrm{F}(T)$ which is nearest to $u$. To see this, let $p$ be any fixed point of $T$. For $t \in(0,1)$, by Proposition 3.1 (2), we have

$$
\cos d(u, p) \leq \cos d\left(u, x_{t}\right) \cos d\left(x_{t}, p\right) .
$$

Letting $t \rightarrow 0^{+}$yields

$$
\cos d(u, p) \leq \cos d(u, q) \cos d(q, p) .
$$

Hence, the conclusion follows from Proposition 2.2.

Finally, we will prove the statement (2). Let $\left\{x_{t_{k}}\right\}$ be a sequence given by the formula (3.1) with $\lim _{k \rightarrow \infty} t_{k}=0$. Then the sequence $\left\{x_{t_{k}}\right\}$ converges to $q:=P_{\mathrm{F}(T)} u$. Let $\mu$ be a Banach limit and $\left\{y_{n}\right\}$ be 
a sequence in $X$ such that $\lim _{n \rightarrow \infty} d\left(y_{n}, T y_{n}\right)=0$. If $u \in \mathrm{F}(T)$, then $x_{t}=u$ for all $t \in(0,1)$ and the result follows. On the other hand, if $u \notin \mathrm{F}(T)$, then $T x_{t} \neq u$ for all $t \in(0,1)$. By Lemma 2.1 , we have

$$
\begin{aligned}
\cos d\left(y_{n}, x_{t_{k}}\right)= & \cos d\left(y_{n}, t_{k} u \oplus\left(1-t_{k}\right) T x_{t_{k}}\right) \\
\geq & \cos d\left(y_{n}, u\right) \frac{\sin t_{k} d\left(u, T x_{t_{k}}\right)}{\sin d\left(u, T x_{t_{k}}\right)}+\cos d\left(y_{n}, T x_{t_{k}}\right) \frac{\sin \left(1-t_{k}\right) d\left(u, T x_{t_{k}}\right)}{\sin d\left(u, T x_{t_{k}}\right)} \\
\geq & \cos d\left(y_{n}, u\right) \frac{\sin t_{k} d\left(u, T x_{t_{k}}\right)}{\sin d\left(u, T x_{t_{k}}\right)} \\
& +\cos \left(d\left(y_{n}, T y_{n}\right)+d\left(T y_{n}, T x_{t_{k}}\right)\right) \frac{\sin \left(1-t_{k}\right) d\left(u, T x_{t_{k}}\right)}{\sin d\left(u, T x_{t_{k}}\right)} \\
\geq & \cos d\left(y_{n}, u\right) \frac{\sin t_{k} d\left(u, T x_{t_{k}}\right)}{\sin d\left(u, T x_{t_{k}}\right)} \\
& +\cos \left(d\left(y_{n}, T y_{n}\right)+d\left(y_{n}, x_{t_{k}}\right)\right) \frac{\sin \left(1-t_{k}\right) d\left(u, T x_{t_{k}}\right)}{\sin d\left(u, T x_{t_{k}}\right)}
\end{aligned}
$$

for all $k, n \in \mathbb{N}$. This implies that

$$
\begin{aligned}
\mu_{n} \cos d\left(y_{n}, x_{t_{k}}\right) \geq & \frac{\sin t_{k} d\left(u, T x_{t_{k}}\right)}{\sin d\left(u, T x_{t_{k}}\right)} \mu_{n} \cos d\left(y_{n}, u\right) \\
& \quad+\frac{\sin \left(1-t_{k}\right) d\left(u, T x_{t_{k}}\right)}{\sin d\left(u, T x_{t_{k}}\right)} \mu_{n} \cos d\left(y_{n}, x_{t_{k}}\right) .
\end{aligned}
$$

Hence, we have

$$
\begin{aligned}
\mu_{n} \cos d\left(y_{n}, x_{t_{k}}\right) & \geq \frac{\sin t_{k} d\left(u, T x_{t_{k}}\right)}{\sin d\left(u, T x_{t_{k}}\right)-\sin \left(1-t_{k}\right) d\left(u, T x_{t_{k}}\right)} \mu_{n} \cos d\left(y_{n}, u\right) \\
& =\frac{\sin t_{k} d\left(u, T x_{t_{k}}\right)}{2 \sin \frac{t_{k}}{2} d\left(u, T x_{t_{k}}\right) \cos \left(1-\frac{t_{k}}{2}\right) d\left(u, T x_{t_{k}}\right)} \mu_{n} \cos d\left(y_{n}, u\right)
\end{aligned}
$$

for all $k \in \mathbb{N}$. Letting $k \rightarrow \infty$ yields

$$
\mu_{n} \cos d\left(y_{n}, q\right) \geq \frac{\mu_{n} \cos d\left(y_{n}, u\right)}{\cos d(u, q)}
$$

Consequently, we have $\cos d(u, q) \geq \mu_{n} \cos d\left(y_{n}, u\right)$, and the proof is finished.

Let $S, T_{n}: X \rightarrow X$ be nonexpansive mappings for all $n \in \mathbb{N}$. Recall that $\left(\left\{T_{n}\right\}, S\right)$ satisfies the NST-condition [24] if $\mathrm{F}(S)=\bigcap_{n=1}^{\infty} \mathrm{F}\left(T_{n}\right)$ and

$$
\lim _{n \rightarrow \infty} d\left(x_{n}, T_{n} x_{n}\right)=0 \text { implies } \lim _{n \rightarrow \infty} d\left(x_{n}, S x_{n}\right)=0
$$

for any sequence $\left\{x_{n}\right\}$ in $X$.

Next, we will prove Browder's type convergence theorem for a family of nonexpansive mappings.

Theorem 3.4. Let $S, T_{n}: X \rightarrow X$ be nonexpansive mappings for all $n \in \mathbb{N}$. Let $u \in X$ be fixed and $\left\{x_{n}\right\}$ be a sequence in $X$ defined by

$$
x_{n}=\alpha_{n} u \oplus\left(1-\alpha_{n}\right) T_{n} x_{n} \quad \text { for } n \in \mathbb{N},
$$

where $\left\{\alpha_{n}\right\}$ is a sequence in $(0,1)$ such that $\lim _{n \rightarrow \infty} \alpha_{n}=0$. If $\left(\left\{T_{n}\right\}, S\right)$ satisfies the NST-condition with $\mathrm{F}(S)=\bigcap_{n=1}^{\infty} \mathrm{F}\left(T_{n}\right) \neq \varnothing$, then $\left\{x_{n}\right\}$ converges strongly to the point $P_{\mathrm{F}(S)} u$. 
Proof. Note that $\mathrm{F}(S)$ is closed and convex. We have $d\left(x_{n}, T_{n} x_{n}\right)=\alpha_{n} d\left(u, T_{n} x_{n}\right) \rightarrow 0$ as $n \rightarrow \infty$. Then the NST-condition asserts that

$$
\lim _{n \rightarrow \infty} d\left(x_{n}, S x_{n}\right)=0
$$

Let $\left\{z_{t}\right\}$ be a net in $X$ defined by

$$
z_{t}=t u \oplus(1-t) S z_{t} \quad \text { for } t \in(0,1) .
$$

By Theorem 3.3, it follows that $\left\{z_{t}\right\}$ converges strongly to $\widehat{z}=P_{\mathrm{F}(S)} u$ as $t \rightarrow 0^{+}$. If $u \in \mathrm{F}(S)$, then we are done. On the other hand, if $u \notin \mathrm{F}(S)$, then for given points $n \in \mathbb{N}$ and $t \in(0,1)$, it follows from Lemma 2.1 that

$$
\begin{aligned}
& \cos d\left(x_{n}, z_{t}\right) \sin d\left(u, S z_{t}\right) \\
\geq & \cos d\left(x_{n}, u\right) \sin t d\left(u, S z_{t}\right)+\cos d\left(x_{n}, S z_{t}\right) \sin (1-t) d\left(u, S z_{t}\right) \\
\geq & \cos d\left(x_{n}, u\right) \sin t d\left(u, S z_{t}\right)+\cos \left(d\left(x_{n}, S x_{n}\right)+d\left(S x_{n}, S z_{t}\right)\right) \sin (1-t) d\left(u, S z_{t}\right) \\
\geq & \cos d\left(x_{n}, u\right) \sin t d\left(u, S z_{t}\right)+\cos \left(d\left(x_{n}, S x_{n}\right)+d\left(x_{n}, z_{t}\right)\right) \sin (1-t) d\left(u, S z_{t}\right) \\
= & \cos d\left(x_{n}, u\right) \sin t d\left(u, S z_{t}\right)+\cos d\left(x_{n}, S x_{n}\right) \cos d\left(x_{n}, z_{t}\right) \sin (1-t) d\left(u, S z_{t}\right) \\
& -\sin d\left(x_{n}, S x_{n}\right) \sin d\left(x_{n}, z_{t}\right) \sin (1-t) d\left(u, S z_{t}\right),
\end{aligned}
$$

and thus

$$
\begin{aligned}
& \cos d\left(x_{n}, z_{t}\right) \\
& \geq \frac{\cos d\left(x_{n}, u\right) \sin t d\left(u, S z_{t}\right)-\sin d\left(x_{n}, S x_{n}\right) \sin d\left(x_{n}, z_{t}\right) \sin (1-t) d\left(u, S z_{t}\right)}{\sin d\left(u, S z_{t}\right)-\cos d\left(x_{n}, S x_{n}\right) \sin (1-t) d\left(u, S z_{t}\right)} .
\end{aligned}
$$

Taking the limit inferior to both sides of the above inequality as $n \rightarrow \infty$, we obtain

$$
\begin{aligned}
& \liminf _{n \rightarrow \infty} \cos d\left(x_{n}, z_{t}\right) \\
& \geq \frac{\sin t d\left(u, S z_{t}\right)}{\left(\sin d\left(u, S z_{t}\right)-\sin (1-t) d\left(u, S z_{t}\right)\right)} \liminf _{n \rightarrow \infty} \cos d\left(x_{n}, u\right) \\
& =\frac{\sin t d\left(u, S z_{t}\right)}{2 \sin (t / 2) d\left(u, S z_{t}\right) \cos (1-t / 2) d\left(u, S z_{t}\right)} \liminf _{n \rightarrow \infty} \cos d\left(x_{n}, u\right) .
\end{aligned}
$$

Taking the limit as $t \rightarrow 0^{+}$yields

$$
\liminf _{n \rightarrow \infty} \cos d\left(x_{n}, \widehat{x}\right) \geq \liminf _{n \rightarrow \infty} \frac{\cos d\left(x_{n}, u\right)}{\cos d(u, \widehat{x})} .
$$

Note that $\cos d(u, \widehat{x}) \leq \cos d\left(u, x_{n}\right) \cos d\left(x_{n}, \widehat{x}\right)$ for all $n \in \mathbb{N}$ by Proposition 3.1. This implies that

$$
\liminf _{n \rightarrow \infty} \cos d\left(x_{n}, \widehat{x}\right) \geq 1
$$

As a result, the sequence $\left\{x_{n}\right\}$ converges strongly to the point $\widehat{x}=P_{\mathrm{F}(S)} u$.

Now, we can extend the above theorem to viscosity approximations. To be precise, the following theorem yields an implication of the Browder's type convergence theorem on viscosity approximations in CAT(1) spaces.

Theorem 3.5. Let $X, S,\left\{T_{n}\right\}$ be as in the preceding theorem and $f: X \rightarrow X$ be a contraction with a constant k. Define a sequence $\left\{x_{n}\right\}$ in $X$ by

$$
x_{n}=\alpha_{n} f\left(x_{n}\right) \oplus\left(1-\alpha_{n}\right) T_{n} x_{n} \quad \text { for all } n \in \mathbb{N},
$$


where $\left\{\alpha_{n}\right\}$ is a sequence in $(0,1)$ such that $\lim _{n \rightarrow \infty} \alpha_{n}=0$. Suppose that $M<\pi / 2$ and

$$
k<\frac{4 \sin ^{2} \frac{M}{2} \cos M}{M^{2}} .
$$

If $\left(\left\{T_{n}\right\}, S\right)$ satisfies the NST-condition with $\mathrm{F}:=\bigcap_{n=1}^{\infty} \mathrm{F}\left(T_{n}\right)=\mathrm{F}(S) \neq \varnothing$, then the sequence $\left\{x_{n}\right\}$ converges strongly to the point $p=P_{F} f(p)$.

Proof. By Lemma 2.4, we know that $P_{F} \circ f$ is a contraction, so there exists a unique fixed point $p$ of $P_{F} \circ f$. Define a sequence $\left\{y_{n}\right\}$ by

$$
y_{n}=\alpha_{n} f(p) \oplus\left(1-\alpha_{n}\right) T_{n} y_{n} \quad \text { for all } n \in \mathbb{N}
$$

The strong convergence of $\left\{y_{n}\right\}$ to $p=P_{F} f(p)$ is assured by Theorem 3.4. For $n \in \mathbb{N}$, it follows from Lemma 2.5 that

$$
\begin{aligned}
d & \left(x_{n}, y_{n}\right) \\
\leq & d\left(x_{n}, \alpha_{n} f(p) \oplus\left(1-\alpha_{n}\right) T_{n} x_{n}\right)+d\left(\alpha_{n} f(p) \oplus\left(1-\alpha_{n}\right) T_{n} x_{n}, y_{n}\right) \\
\leq & \frac{\sin \alpha_{n} M}{\sin M} d\left(f\left(x_{n}\right), f(p)\right)+\frac{\sin \left(1-\alpha_{n}\right) M}{\sin M} d\left(f\left(x_{n}\right), f\left(y_{n}\right)\right) \\
\leq & \frac{\sin \alpha_{n} M}{\sin M}\left(d\left(f\left(x_{n}\right), f\left(y_{n}\right)\right)+d\left(f\left(y_{n}\right), f(p)\right)+\frac{\sin \left(1-\alpha_{n}\right) M}{\sin M} d\left(f\left(x_{n}\right), f\left(y_{n}\right)\right)\right. \\
\leq & \frac{\sin \alpha_{n} M}{\sin M} d\left(y_{n}, p\right)+\left(k \frac{\sin \alpha_{n} M}{\sin M}+\frac{\sin \left(1-\alpha_{n}\right) M}{\sin M}\right) d\left(x_{n}, y_{n}\right) \\
= & \left(1-\left(1-k \frac{\sin \alpha_{n} M}{\sin M}-\frac{\sin \left(1-\alpha_{n}\right) M}{\sin M}\right)\right) d\left(x_{n}, y_{n}\right)+\frac{\sin \alpha_{n} M}{\sin M} d\left(y_{n}, p\right) \\
= & \left(1-\frac{\alpha_{n} M}{\sin M}\left(\frac{\sin M-\sin \left(1-\alpha_{n}\right) M}{\alpha_{n} M}-k \frac{\sin \alpha_{n} M}{\alpha_{n} M}\right)\right) d\left(x_{n}, y_{n}\right) \\
& +\frac{\alpha_{n} M}{\sin M}\left(\frac{\sin \alpha_{n} M}{\alpha_{n} M} d\left(y_{n}, p\right)\right),
\end{aligned}
$$

which implies that

$$
\left(\frac{\sin M-\sin \left(1-\alpha_{n}\right) M}{\sin \alpha_{n} M}-k\right) d\left(x_{n}, y_{n}\right) \leq d\left(y_{n}, p\right) .
$$

Notice that $k<\cos M$. Hence, we have

$$
\lim _{n \rightarrow \infty}\left(\frac{\sin M-\sin \left(1-\alpha_{n}\right) M}{\sin \alpha_{n} M}-k\right)=\cos M-k>0 .
$$

Consequently, it follows that $\lim _{n \rightarrow \infty} d\left(x_{n}, y_{n}\right)=0$, and the proof is finished.

\section{Strong convergence theorems for Halpern's type}

Throughout this section, we define the following conditions:

(C1) $\lim _{n \rightarrow \infty} \alpha_{n}=0$ and $\sum_{n=1}^{\infty} \alpha_{n}=\infty$;

(C2) $\sum_{n=1}^{\infty}\left|\alpha_{n}-\alpha_{n+1}\right|<\infty$ or $\lim _{n \rightarrow \infty} \frac{\alpha_{n+1}}{\alpha_{n}}=1$;

$\left(\mathrm{C} 2^{\prime}\right) \sum_{n=1}^{\infty}\left|\alpha_{n}-\alpha_{n+1}\right|<\infty$ or $\lim _{n \rightarrow \infty} \frac{\alpha_{n}-\alpha_{n+1}}{\alpha_{n}^{2}}=0$. 
Let $\left\{T_{n}\right\}$ be a sequence of nonexpansive mappings on $X$ with a common fixed point, and $\left\{\alpha_{n}\right\}$ be a sequence in $(0,1)$ satisfying the condition (C1). We say that $\left(X,\left\{T_{n}\right\},\left\{\alpha_{n}\right\}\right)$ has the Halpern property [25] if for each $u \in X$, a sequence

$$
\left\{x_{n}\right\}:=\left\{\begin{array}{l}
x_{1} \in X \\
x_{n+1}=\alpha_{n} u \oplus\left(1-\alpha_{n}\right) T_{n} x_{n} \quad(\text { for } n \in \mathbb{N})
\end{array}\right.
$$

converges strongly.

We first discuss Halpern's type convergence.

Proposition 4.1. Suppose that $\left(X,\left\{T_{n}\right\},\left\{\alpha_{n}\right\}\right)$ has the Halpern property. For each $u \in X$, define a mapping $P: X \rightarrow X$ by

$$
P u:=\lim _{n \rightarrow \infty} x_{n},
$$

where $\left\{x_{n}\right\}$ is the sequence defined by (4). Suppose, in addition, that $M<\pi / 2$ or $\sum_{n=1}^{\infty} \alpha_{n}^{2}=\infty$. Then $P$ is well defined, that is, it is independent of the choice of the initial $x_{1}$.

Proof. Fix $x_{1}=u \in X$ and let $y_{1}$ be an arbitrary element in $X$. Define sequences $\left\{x_{n}\right\}$ and $\left\{y_{n}\right\}$ in $X$ by

$$
x_{n+1}=\alpha_{n} u \oplus\left(1-\alpha_{n}\right) T_{n} x_{n} \text { and } y_{n+1}=\alpha_{n} u \oplus\left(1-\alpha_{n}\right) T_{n} y_{n} \quad \text { for } n \in \mathbb{N} .
$$

For each $n \in \mathbb{N}$, let

$$
\beta_{n}:=1-\frac{\sin \left(1-\alpha_{n}\right) M}{\sin M}
$$

It follows from Lemma 2.5 that

$$
d\left(x_{n+1}, y_{n+1}\right) \leq \frac{\sin \left(1-\alpha_{n}\right) M}{\sin M} d\left(T_{n} x_{n}, T_{n} y_{n}\right) \leq(1-\beta) d\left(x_{n}, y_{n}\right) .
$$

To apply Lemma 2.7 , it suffices to show that $\sum_{n=1}^{\infty} \beta_{n}=\infty$. Assume first that $M<\pi / 2$. Then

$$
\begin{aligned}
\beta_{n} & =\frac{2}{\sin M} \sin \left(\frac{\alpha_{n}}{2} M\right) \cos \left(\left(1-\frac{\alpha_{n}}{2}\right) M\right) \\
& \geq \alpha_{n} \cos \left(\left(1-\frac{\alpha_{n}}{2}\right) M\right) \\
& \geq \alpha_{n} \cos M .
\end{aligned}
$$

Since $\sum_{n=1}^{\infty} \alpha_{n}=\infty$, we have $\sum_{n=1}^{\infty} \beta_{n}=\infty$. On the other hand, assume that $\sum_{n=1}^{\infty} \alpha_{n}^{2}=\infty$. Note that

$$
\begin{aligned}
\beta_{n} & \geq 1-\sin \left(\frac{\left(1-\alpha_{n}\right) \pi}{2}\right) \\
& =\sin ^{2}\left(\frac{\alpha_{n} \pi}{4}\right) \\
& \geq \alpha_{n}^{2} \sin ^{2}\left(\frac{\pi}{4}\right) .
\end{aligned}
$$

Thus, we obtain $\sum_{n=1}^{\infty} \beta_{n}=\infty$. By Lemma 2.7, we have $\lim _{n \rightarrow \infty} d\left(x_{n}, y_{n}\right)=0$, and hence $P u$ is well defined.

Recall that a countable family of nonexpansive mappings $\left\{T_{n}: X \rightarrow X\right\}$ satisfies the AKTT-condition [26] if $\sum_{n=1}^{\infty} \sup \left\{d\left(T_{n+1} x, T_{n} x\right): x \in X\right\}<\infty$. In this case, it follows that $S x:=$ $\lim _{n \rightarrow \infty} T_{n} x$ exists for all $x \in X$, and we also say that $\left(\left\{T_{n}\right\}, S\right)$ satisfies the AKTT-condition.

We are now ready to prove the Halpern's type convergence theorem for a family of nonexpansive mappings. 
Theorem 4.2. Let $S, T_{n}: X \rightarrow X$ be nonexpansive mappings for all $n \in \mathbb{N}$ such that $\left(\left\{T_{n}\right\}, S\right)$ satisfies the AKTT-condition with $\mathrm{F}:=\bigcap_{n=1}^{\infty} \mathrm{F}\left(T_{n}\right)=\mathrm{F}(S) \neq \varnothing$. Let $u=x_{1} \in X$ be fixed and $\left\{x_{n}\right\}$ be a sequence in $X$ defined by

$$
x_{n+1}=\alpha_{n} u \oplus\left(1-\alpha_{n}\right) T_{n} x_{n} \quad \text { for all } n \in \mathbb{N},
$$

where $\left\{\alpha_{n}\right\}$ is a sequence in $(0,1)$ satisfying the condition (C1). Suppose that one of the following conditions holds:

(M1) The sequence $\left\{\alpha_{n}\right\}$ satisfies the condition (C2) and $M<\pi / 2$.

(M2) The sequence $\left\{\alpha_{n}\right\}$ satisfies the condition $\left(\mathrm{C} 2^{\prime}\right)$ and $\sum_{n=1}^{\infty} \alpha_{n}^{2}=\infty$.

Then $\left\{x_{n}\right\}$ converges strongly to the point $P_{\mathrm{F}} u$.

Proof. For each $n \in \mathbb{N}$, let

$$
\beta_{n}:=1-\frac{\sin \left(1-\alpha_{n}\right) M}{\sin M} .
$$

In the same way as in the proof of Proposition 4.1, we have $\sum_{n=1}^{\infty} \beta_{n}=\infty$. By Lemma 2.5, we obtain

$$
\begin{aligned}
d\left(x_{n+1}, x_{n}\right) \leq & d\left(\alpha_{n} u \oplus\left(1-\alpha_{n}\right) T_{n} x_{n}, \alpha_{n} u \oplus\left(1-\alpha_{n}\right) T_{n} x_{n-1}\right) \\
& +d\left(\alpha_{n} u \oplus\left(1-\alpha_{n}\right) T_{n} x_{n-1}, \alpha_{n} u \oplus\left(1-\alpha_{n}\right) T_{n-1} x_{n-1}\right) \\
& +d\left(\alpha_{n} u \oplus\left(1-\alpha_{n}\right) T_{n-1} x_{n-1}, \alpha_{n-1} u \oplus\left(1-\alpha_{n-1}\right) T_{n-1} x_{n-1}\right) \\
\leq & \frac{\sin \left(1-\alpha_{n}\right) M}{\sin M} d\left(T_{n} x_{n-1}, T_{n} x_{n}\right)+d\left(T_{n-1} x_{n-1}, T_{n} x_{n-1}\right) \\
& +\left|\alpha_{n}-\alpha_{n+1}\right| d\left(u, T_{n-1} x_{n-1}\right) \\
\leq & \left(1-\beta_{n}\right) d\left(x_{n-1}, x_{n}\right)+\sup _{z \in\left\{x_{n}\right\}} d\left(T_{n} z, T_{n-1} z\right)+\left|\alpha_{n}-\alpha_{n+1}\right| M \\
= & \left(1-\beta_{n}\right) d\left(x_{n-1}, x_{n}\right)+\sup _{z \in\left\{x_{n}\right\}} d\left(T_{n} z, T_{n-1} z\right)+\beta_{n}\left(\frac{\alpha_{n}}{\beta_{n}}\right)\left|1-\frac{\alpha_{n+1}}{\alpha_{n}}\right| M \\
= & \left(1-\beta_{n}\right) d\left(x_{n-1}, x_{n}\right)+\sup _{z \in\left\{x_{n}\right\}} d\left(T_{n} z, T_{n-1} z\right)+\beta_{n}\left(\frac{\alpha_{n}^{2}}{\beta_{n}}\right)\left|\frac{\alpha_{n}-\alpha_{n+1}}{\alpha_{n}^{2}}\right| M
\end{aligned}
$$

for all $n \in \mathbb{N}$. Note that if $M<\pi / 2$, then

$$
\lim _{n \rightarrow \infty} \frac{\alpha_{n}}{\beta_{n}}=\lim _{n \rightarrow \infty} \frac{\alpha_{n} \sin M}{2 \sin \left(\frac{\alpha_{n}}{2} M\right) \cos \left(\left(1-\frac{\alpha_{n}}{2}\right) M\right)}=\frac{\sin M}{M \cos M}
$$

On the other hand, if $M=\pi / 2$, then

$$
\lim _{n \rightarrow \infty} \frac{\alpha_{n}^{2}}{\beta_{n}}=\lim _{n \rightarrow \infty} \frac{\alpha_{n}^{2}}{\sin ^{2}\left(\frac{\alpha_{n} \pi}{4}\right)}=\frac{16}{\pi^{2}} .
$$

Therefore, we can apply Lemma 2.7 with the conditions (M1) or (M2) to obtain

$$
\lim _{n \rightarrow \infty} d\left(x_{n+1}, x_{n}\right)=0
$$


This implies that $\lim _{n \rightarrow \infty} d\left(T_{n} x_{n}, x_{n}\right)=0$, and hence

$$
\begin{aligned}
\lim _{n \rightarrow \infty} d\left(x_{n}, S x_{n}\right) & \leq \lim _{n \rightarrow \infty}\left(d\left(x_{n}, T_{n} x_{n}\right)+d\left(T_{n} x_{n}, S x_{n}\right)\right) \\
& \leq \lim _{n \rightarrow \infty}\left(d\left(x_{n}, T_{n} x_{n}\right)+\sup _{z \in\left\{x_{n}\right\}} d\left(T_{n} z, S z\right)\right) \\
& \leq \lim _{n \rightarrow \infty}\left(d\left(x_{n}, T_{n} x_{n}\right)+\sum_{k=n}^{\infty} \sup _{z \in\left\{x_{n}\right\}} d\left(T_{k} z, T_{k+1} z\right)\right) \\
& =0 .
\end{aligned}
$$

Let $\left\{x_{t}\right\}$ be given by the formula (3.1), that is,

$$
x_{t}=t u \oplus(1-t) S x_{t}
$$

for all $t \in(0,1)$. By Theorem 3.3, we know that $p:=\lim _{t \rightarrow 0^{+}} x_{t}$ is the point of $\mathrm{F}(S)$ which is nearest to $u$. For each $n \in \mathbb{N}$, let

$$
\begin{aligned}
& a_{n}:=\sin ^{2} \frac{d\left(x_{n}, p\right)}{2}, \\
& b_{n}:=1-\frac{\cos d(u, p)}{\sin d\left(u, T_{n} x_{n}\right) \tan \frac{\alpha_{n}}{2} d\left(u, T_{n} x_{n}\right)+\cos d\left(u, T_{n} x_{n}\right)} .
\end{aligned}
$$

In fact, we obtain from Lemma 3.1 in [7] that

$$
a_{n+1} \leq\left(1-\beta_{n}\right) a_{n}+\beta_{n} b_{n}
$$

for all $n \in \mathbb{N}$. Since $\lim _{n \rightarrow \infty} d\left(x_{n+1}, x_{n}\right)=0$, we have

$$
\begin{aligned}
& \limsup _{n \rightarrow \infty}\left(\left(\cos d\left(u, x_{n}\right)-\cos d(u, p)\right)-\left(\cos d\left(u, x_{n+1}\right)-\cos d(u, p)\right)\right) \\
& =\limsup _{n \rightarrow \infty}\left(\cos d\left(u, x_{n}\right)-\cos d\left(u, x_{n+1}\right)\right) \\
& =2 \limsup _{n \rightarrow \infty} \sin \left(\frac{d\left(u, x_{n}\right)+d\left(u, x_{n+1}\right)}{2}\right) \sin \left(\frac{d\left(u, x_{n+1}\right)-d\left(u, x_{n}\right)}{2}\right) \\
& =0 .
\end{aligned}
$$

By Theorem 3.3, we have $\mu_{n}\left(\cos d\left(u, x_{n}\right)-\cos d(u, p)\right) \leq 0$ for all Banach limits $\mu$. It follows from Lemma 2.8 that

$$
\limsup _{n \rightarrow \infty}\left(\cos d\left(u, x_{n}\right)-\cos d(u, p)\right) \leq 0 .
$$

Note that $\lim _{n \rightarrow \infty} d\left(x_{n}, T_{n} x_{n}\right)=0$. Consequently, we have

$$
\begin{aligned}
\limsup _{n \rightarrow \infty} b_{n} & =\limsup _{n \rightarrow \infty}\left(1-\frac{\cos d(u, p)}{\sin d\left(u, T_{n} x_{n}\right) \tan \frac{\alpha_{n}}{2} d\left(u, T_{n} x_{n}\right)+\cos d\left(u, T_{n} x_{n}\right)}\right) \\
& =\limsup _{n \rightarrow \infty}\left(1-\frac{\cos d(u, p)}{\cos d\left(u, x_{n}\right)}\right) \\
& \leq 0
\end{aligned}
$$

Finally, Lemma 2.7 guarantees the strong convergence of $\left\{x_{n}\right\}$ to $p$, which is the desired result. 
Finally, we will discuss viscosity approximations for a family of nonexpansive mappings using the above theorem. To be precise, the following theorem show that the Halpern's type convergence theorem implies viscosity approximations in CAT(1) spaces.

Theorem 4.3. Let $X, S,\left\{T_{n}\right\}$ be as in the preceding theorem and $f: X \rightarrow X$ be a contraction with a constant $k$. Let $\left\{x_{n}\right\}$ be a sequence in $X$ defined by $x_{1} \in X$ and

$$
x_{n+1}=\alpha_{n} f\left(x_{n}\right) \oplus\left(1-\alpha_{n}\right) T_{n} x_{n} \quad \text { for } n \in \mathbb{N},
$$

where $\left\{\alpha_{n}\right\}$ is a sequence in $(0,1)$ satisfying the conditions (C1) and (C2). Suppose that $M<\pi / 2$ and

$$
k<\frac{4 \sin ^{2} \frac{M}{2} \cos M}{M^{2}}
$$

Then $\left\{x_{n}\right\}$ converges strongly to the point $p=P_{F} f(p)$.

Proof. By Lemma 2.4, we know that $P_{F} \circ f$ is a contraction, so there exists a unique fixed point $p$ of $P_{F} \circ f$. Define a sequence $\left\{y_{n}\right\}$ in $X$ by $y_{1}=f(p)$ and

$$
y_{n+1}=\alpha_{n} f(p) \oplus\left(1-\alpha_{n}\right) T_{n} y_{n} \quad \text { for all } n \in \mathbb{N} .
$$

Hence, the strong convergence of $\left\{y_{n}\right\}$ to $P_{F} f(p)$ is assured by Theorem 4.2. Applying Lemma 2.5, we obtain

$$
\begin{aligned}
d & \left(x_{n+1}, y_{n+1}\right) \\
\leq & d\left(x_{n+1}, \alpha_{n} f(p) \oplus\left(1-\alpha_{n}\right) T_{n} x_{n}\right)+d\left(\alpha_{n} f(p) \oplus\left(1-\alpha_{n}\right) T_{n} x_{n}, y_{n+1}\right) \\
\leq & \frac{\sin \alpha_{n} M}{\sin M} d\left(f\left(x_{n}\right), f(p)\right)+\frac{\sin \left(1-\alpha_{n}\right) M}{\sin M} d\left(f\left(x_{n}\right), f\left(y_{n}\right)\right) \\
\leq & \frac{\sin \alpha_{n} M}{\sin M}\left(d\left(f\left(x_{n}\right), f\left(y_{n}\right)\right)+d\left(f\left(y_{n}\right), f(p)\right)+\frac{\sin \left(1-\alpha_{n}\right) M}{\sin M} d\left(f\left(x_{n}\right), f\left(y_{n}\right)\right)\right. \\
\leq & \frac{\sin \alpha_{n} M}{\sin M} d\left(y_{n}, p\right)+\left(k \frac{\sin \alpha_{n} M}{\sin M}+\frac{\sin \left(1-\alpha_{n}\right) M}{\sin M}\right) d\left(x_{n}, y_{n}\right) \\
= & \left(1-\left(1-k \frac{\sin \alpha_{n} M}{\sin M}-\frac{\sin \left(1-\alpha_{n}\right) M}{\sin M}\right)\right) d\left(x_{n}, y_{n}\right)+\frac{\sin \alpha_{n} M}{\sin M} d\left(y_{n}, p\right) \\
= & \left(1-\frac{\alpha_{n} M}{\sin M}\left(\frac{\sin M-\sin \left(1-\alpha_{n}\right) M}{\alpha_{n} M}-k \frac{\sin \alpha_{n} M}{\alpha_{n} M}\right)\right) d\left(x_{n}, y_{n}\right) \\
& +\frac{\alpha_{n} M}{\sin M}\left(\frac{\sin \alpha_{n} M}{\alpha_{n} M} d\left(y_{n}, p\right)\right)
\end{aligned}
$$

for all $n \in \mathbb{N}$. Since $k<\cos M$, we have

$$
\begin{aligned}
& \lim _{n \rightarrow \infty}\left(\frac{\sin M-\sin \left(1-\alpha_{n}\right) M}{\alpha_{n} M}-k \frac{\sin \alpha_{n} M}{\alpha_{n} M}\right) \\
& =\lim _{n \rightarrow \infty}\left(\frac{2 \sin \frac{\alpha_{n} M}{2} \cos \left(1-\alpha_{n} / 2\right) M}{\alpha_{n} M}-k \frac{\sin \alpha_{n} M}{\alpha_{n} M}\right) \\
& =\cos M-k>0 .
\end{aligned}
$$

Hence, Lemma 2.7 guarantees that $\lim _{n \rightarrow \infty} d\left(x_{n}, y_{n}\right)=0$, and this completes the proof. 


\section{Conclusions}

In this paper, we present an existence and convergence theorem for a nonexpansive mapping on a complete CAT(1) space. Furthermore, we prove a strong convergent theorem for Browder's type iterations of a family of nonexpansive mappings. We also show that the Browder's type convergence theorem implies viscosity approximations. Next, we derive our results with Halpern's type iteration of a family of nonexpansive mappings. Finally, Moudafi's viscosity type methods are also discussed in the framework of the space without the nice projection property.

Funding: The Thailand Research Fund under Grant MRG6080243.

Acknowledgments: This research is also supported by Faculty of Science, Khon Kaen University.

Conflicts of Interest: The author declares no conflicts of interest.

\section{References}

1. Kirk, W.A. Geodesic geometry and fixed point theory, in: Seminar of Mathematical Analysis (Malaga/Seville, 2002/2003), in: Colecc. Abierta, 64 Univ. Sevilla Secr. Publ., Seville, 2003, 195-225.

2. Kirk, W.A. Geodesic geometry and fixed point theory II, International Conference on Fixed Point Theory and Applications. Yokohama Publ., Yokohama, 2004, 113-142.

3. Espínola, R.; Fernández-León, A. CAT(k)-spaces, weak convergence and fixed points. J. Math. Anal. Appl. 2009, 353, 410-427.

4. Bačák, M. Convex analysis and optimization in Hadamard spaces. De Gruyter Series in Nonlinear Analysis and Applications, 22. De Gruyter, Berlin, 2014; ISBN: 978-3-11-036103-2; 978-3-11-036162-9.

5. Bridson, M.; Haefliger, A. Metric Spaces of Non-Positive Curvature; Springer: Berlin, Germany, 1999; ISBN 3-540-64324-9.

6. Piątek, B. Halpern iteration in CAT(א) spaces. Acta Math. Sin. (Engl. Ser.) 2011, 27, 635-646.

7. Kimura, Y.; Satô, K. Halpern iteration for strongly quasinonexpansive mappings on a geodesic space with curvature bounded above by one. Fixed Point Theory Appl. 2013, 2013:7, 14 pp.

8. Huang, S. Viscosity approximations with weak contractions in geodesic metric spaces of nonpositive curvature. J. Nonlinear Convex Anal. 2016, 17, 77-91.

9. Shi, L.Y.; Chen, R.D. Strong convergence of viscosity approximation methods for nonexpansive mappings in CAT(0) spaces. J. Appl. Math. 2012, Art. ID 421050, 11 pp.

10. Wangkeeree, R.; Preechasilp, P. Viscosity approximation methods for nonexpansive mappings in CAT $(0)$ spaces. J. Inequal. Appl. 2013, 2013:93, 15 pp.

11. Kaewkhao, A.; Panyanak, B.; Suantai, S. Viscosity iteration method in CAT(0) spaces without the nice projection property. J. Inequal. Appl. 2015, 2015:278, 9 pp.

12. Panyanak, B.; Suantai, S. Viscosity approximation methods for multivalued nonexpansive mappings in geodesic spaces. Fixed Point Theory Appl. 2015, 2015:114, 14 pp.

13. Pakkaranang, N.; Kumam, P; Cho, Y.J.; Saipara, P.; Padcharoen, A.; Khaofong, C. Strong convergence of modified viscosity implicit approximation methods for asymptotically nonexpansive mappings in complete CAT(0) spaces. J. Math. Comput. Sci. 2017, 17, 345-354.

14. Xiong, T.-J.; Lan, H.-Y. Strong convergence of new two-step viscosity iterative approximation methods for set-valued nonexpansive mappings in CAT(0) spaces. J. Funct. Spaces 2018, Art. ID 1280241, 8 pp.

15. Xiong, T.-J.; Lan, H.-Y. General modified viscosity implicit rules for generalized asymptotically nonexpansive mappings in complete CAT(0) spaces. J. Inequal. Appl. 2019, 176, 16 pp.

16. Kimura, Y.; Satô, K. Convergence of subsets of a complete geodesic space with curvature bounded above. Nonlinear Anal. 2012, 75, 5079-5085.

17. Piątek, B.; Viscosity iteration in CAT( $\kappa)$ spaces. Numer. Funct. Anal. Optim. 2013, 34, 1245-1264.

18. Ariza-Ruiz, D.; Fernández-León, A.; López-Acedo, G.; Nicolae, A. Chebyshev sets in geodesic spaces. J. Approx. Theory 2016, 207, 265-282.

19. Kimura, Y.; Kohsaka, F. Spherical nonspreadingness of resolvents of convex functions in geodesic spaces. J. Fixed Point Theory Appl. 2016, 18, 93-115. 
20. Yotkaew, P.; Saejung, S. Strong convergence theorems for multivalued mappings in a geodesic space with curvature bounded above. Fixed Point Theory Appl. 2015, 2015:235, 11 pp.

21. Xu, H.-K. Iterative algorithms for nonlinear operators. J. London Math. Soc. 2002, 66, 240-256.

22. Shioji, N.; Takahashi, W. Strong convergence of approximated sequences for nonexpansive mappings in Banach spaces. Proc. Amer. Math. Soc. 1997, 125, 3641-3645.

23. Saejung, S. Halpern's iteration in CAT(0) spaces. Fixed Point Theory Appl. 2010, Art. ID 471781, 13 pp.

24. Nakajo, K.; Shimoji, K.; Takahashi, W. Strong convergence to common fixed points of families of nonexpansive mappings in Banach spaces. J. Nonlinear Convex Anal. 2007, 8, 11-34.

25. Suzuki, T. Moudafi's viscosity approximations with Meir-Keeler contractions. J. Math. Anal. Appl. 2007, 325, 342-352.

26. Aoyama, K.; Kimura, Y.; Takahashi, W.; Toyoda, M. Approximation of common fixed points of a countable family of nonexpansive mappings in a Banach space. Nonlinear Anal. 2007, 67, 2350-2360. 